Aus der Prov.-Hebeammen-Lehranstslt zu Breslau.

\title{
Retroflexio uteri gravidi partialis; Spontanruptur bei der Geburt ${ }^{1}$ ).
}

Von

Dr. Maiss, Assistenzarzt.

Nachstehender Geburtsfall ist dazu angethan, unser Interesse in mehrfacher Hinsicht wachzurufen und halte ich ihn deshalb der Veröffentlichung werth.

Am Abend des 27. August verflossenen Jahres wurde von einer hiesigen Hebamme eine Kreissende in die Anstalt gebracht mit der Angabe, dass der Kopf nicht eintreten wolle.

Die Kreissende, eine 34 jährige Erstgebärende, ist nie ernstlich krank gewesen; sie will erst mit 3 Jahren laufen gelernt haben. Periode ohne Besonderheiten; letzte Periode Anfang December 1896. In der Schwangerschaft hat Pat. keinerlei Beschwerden gehabt. Die Wehen begannen Tags vorher, also am 26. August Vormittags 11 Ulir, und waren nach Aussage der Hebamme selten und schwach; zu gleicher Zeit sprang die Blase.

Status: Die Kreissende ist eine grosse, gracile Person in mittlerem Ernährungszustande. Temp. 38,0, P. 80, kräftig. Am Knochengerüst keine Zeichen überstandener englischer Krankheit; die Beckenmaasse ergeben keine Abweichung vom Normalen. Der Leib ist mässig ausgedehnt; der Grund der Gebärmutter steht handbreit unterhalb der Brustbeinspitze; ihre Form ist schräg oval; ihre Achse verläuft von rechts oben nach links unten, jhr unterer Pol ist links deutlich vorgebaucht, und man fühlt hier besser wie sonst den auf die linke Darmbeinschaufel abgewichenen Kopf durch. Die Betastung dieser Partie ist schmerzhaft. Contractionsring nicht sichtbar oder deutlich fühlbar. Rücken rechts vorn, kleine Theile links im Grunde. Herztöne rechts unterhalb des Nabels von normaler Frequenz, deutlich hörbar. Wehe» schwach und von langen Pausen unterbrochen.

Da die äussere Untersuchung keinen Grund für das Abweichen des Kopfes erkennen liess, untersuchte man innerlich; man stiess hierbei in

1) Nach einem in der medic. Section der Schles. Gesellsch. f. vaterländ. Cultur am 17. December 1897 gehaltenen Vortrage. 


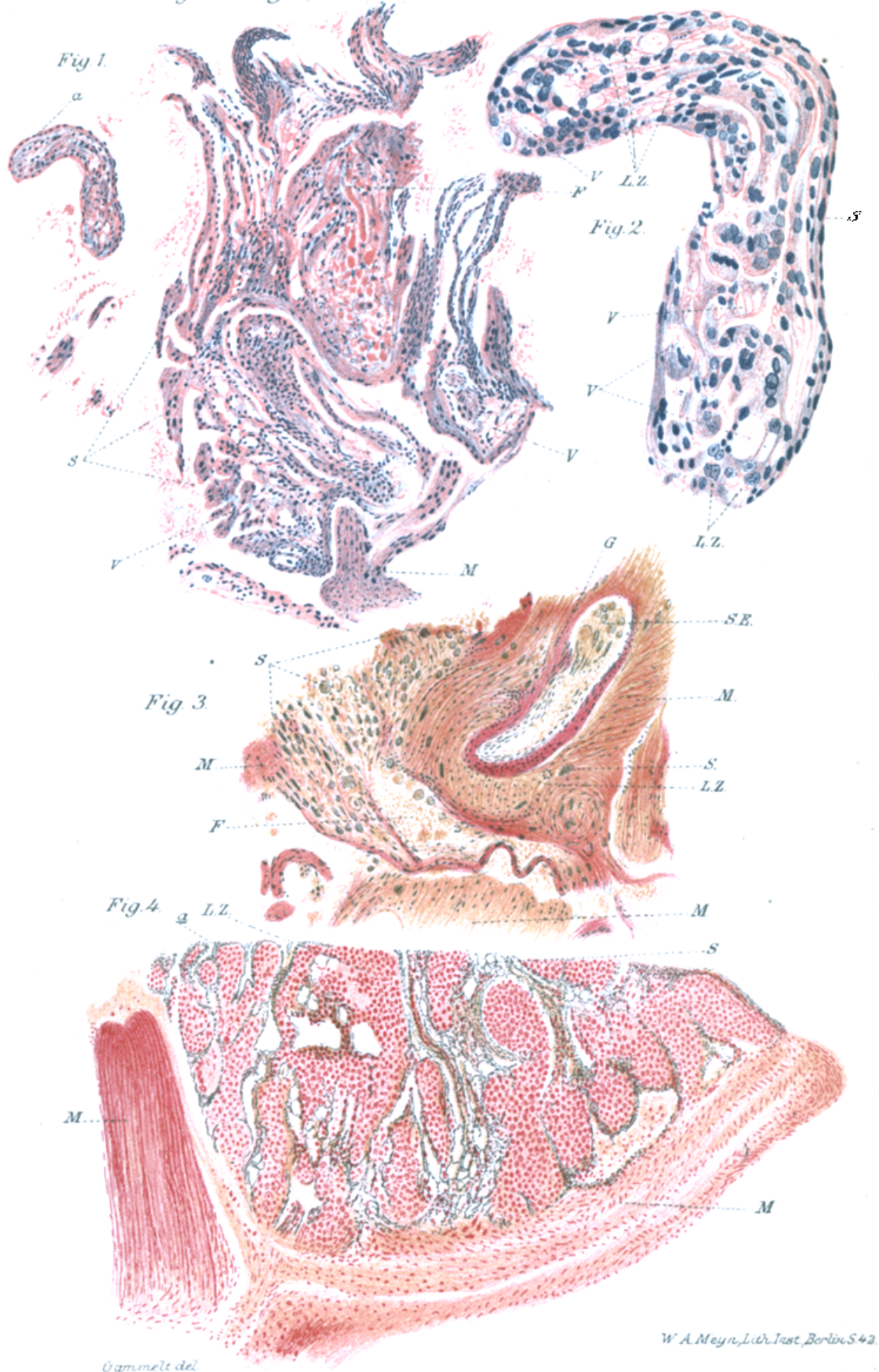




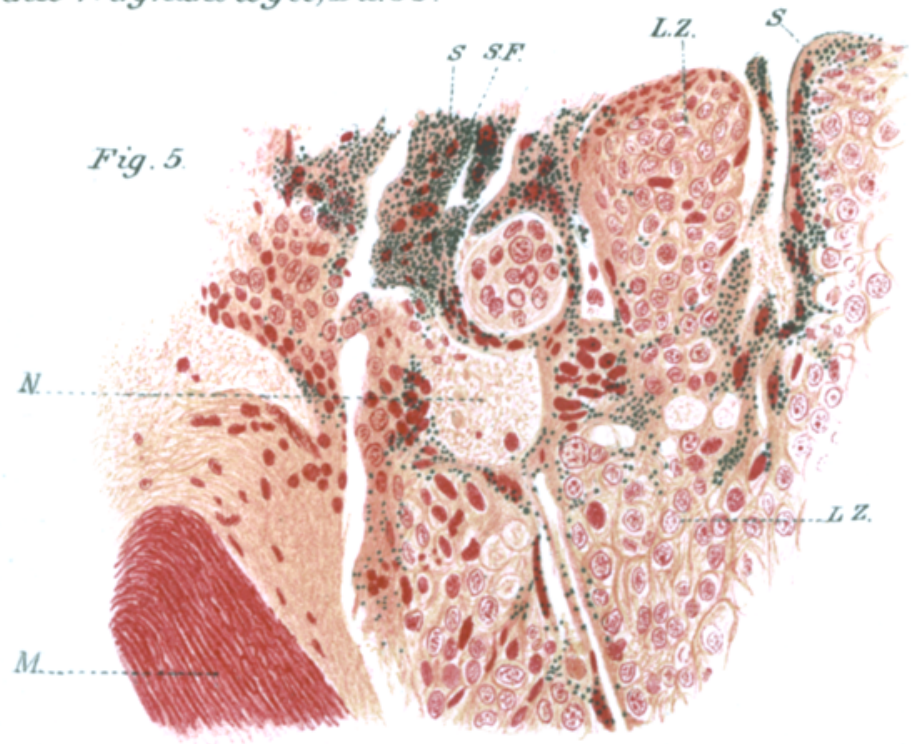

Fig. $A$ ए. $M$

H,W

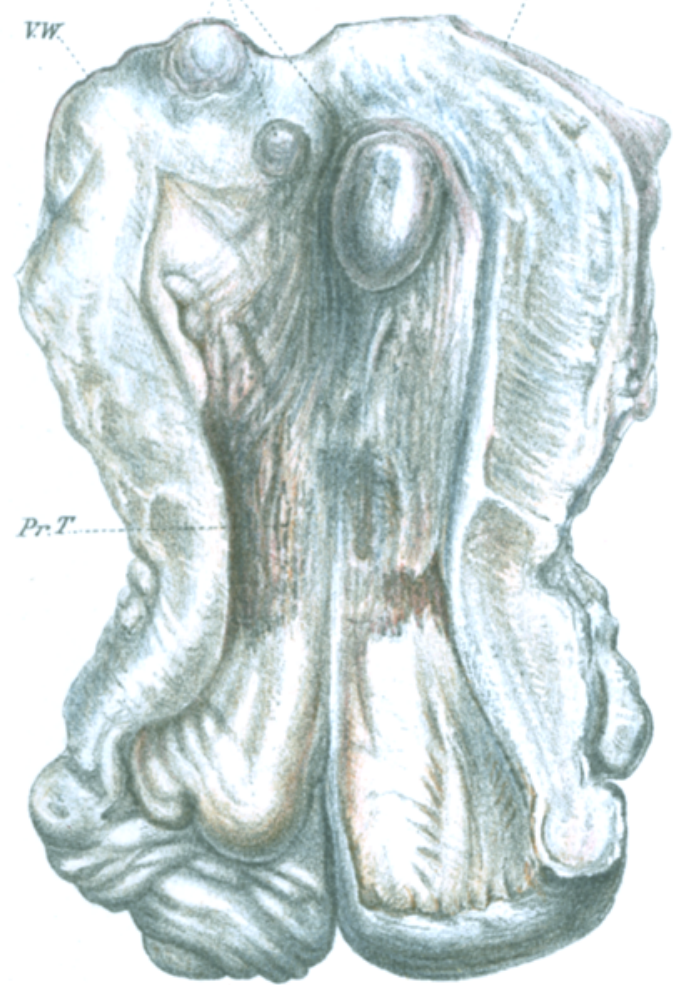

Fig. B.

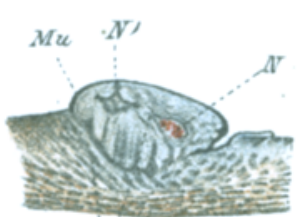

i.

Fig. C.

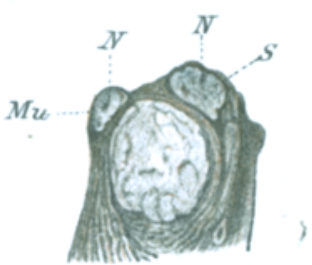


einer Eutfernung von 2 Querfinger rom Scheideneingang a duf cine runde elastische Geschwulst, die sich in das Scheidenlumen halbkugelig vom Beckeneingange her vorwölbte und es ausfüllte; der Muttermund fand sich nach langem Suchen ca. fingerbreit über der Schossfuge, er war thalergross und queroval; die hintere Lippe ging ohne Grenze in erwähnten Tumor über, und bestand kein Zweifel, dass dieser eine Aussackung der binteren Gebärmutterwand vorstellte; hakte man den Finger in den Muttermund ein, so konnte man die hintere Lippe ein Stück nach unten ziehen. Der Yorberg war nicht zu erreichen.

Da lieine dringende Indication zur Geburtsbeendigung weder im Interesse der Mutter, noch des Kindes vorlag, und da man andererseits hoffen konnte, dass, wenn der Kopf eingetreten wäre und auf die hintere Lippe wirken würde, diese sich doch unter kräftigen Wehen über

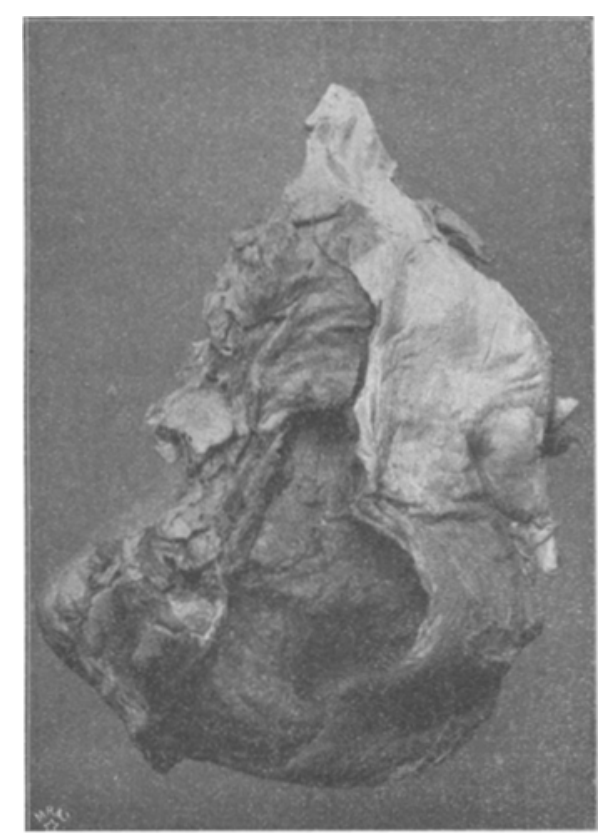

denselben zurückziehen würdc, cino Amahno, die im späteren Geburtsverlaufe ihre Bestätigung fand, so wurde abgewartet. Der Kopf wurde über den Beckeneingang geschoben, der Leib gewickelt und die Frau auf die linke Seite gelagert. Nach diesen Yaassnahmen trat der Kopf in der That unter kräftig einsetzenden Wehen anf den Beckeneingang fest. Als der Stationsarzt Morgens 4 Uhr wegen einer anderen. Kreissenden nach dem Gebärsaal gerufen wurde, war der Kopf mit der kleineren Hälfte ins Becken eingetreten und der Muttermund nahezu vollständig eröffnet. Wehen und Herztöne waren gut. Der Leib der Kreissenden war sehr empfindlich, doch war Contactionsring nieht sichtbar und will der College keine Symptome einer drohenden Ruptur bemerkt haben. 
Gegen $7 \frac{1}{2}$ Lhr Vormittags meldete die Hebamme, dass die Kreissende ganz blau aussähe, und sie ihren Puls nicht fühle. Die Wehen waren in der Zwischenzeit angeblich sehr kräftig und schmerzhaft.

Die Kreissende macht einen collabirten Eindruck. Temperatur 38,8. Puls nicht fühlbar. Fundus steht fast an der Brustbeinspitze; der Kopf zur Hälfte ins Becken eingetreten. Herztöne sind nicht zu hören. Keine Wehen. Die Rreissende klagt über Schmerzen in der linken Seite.

Ueber die Diagnose komnte kein $Z$ weifel sein; es wurde unverzüglich zur Perforation geschritten; vom Mattermund fühlte man vorn und seitwärts einen $1 \mathrm{~cm}$ breiten Saum, die hintere Lippe war verstrichen;

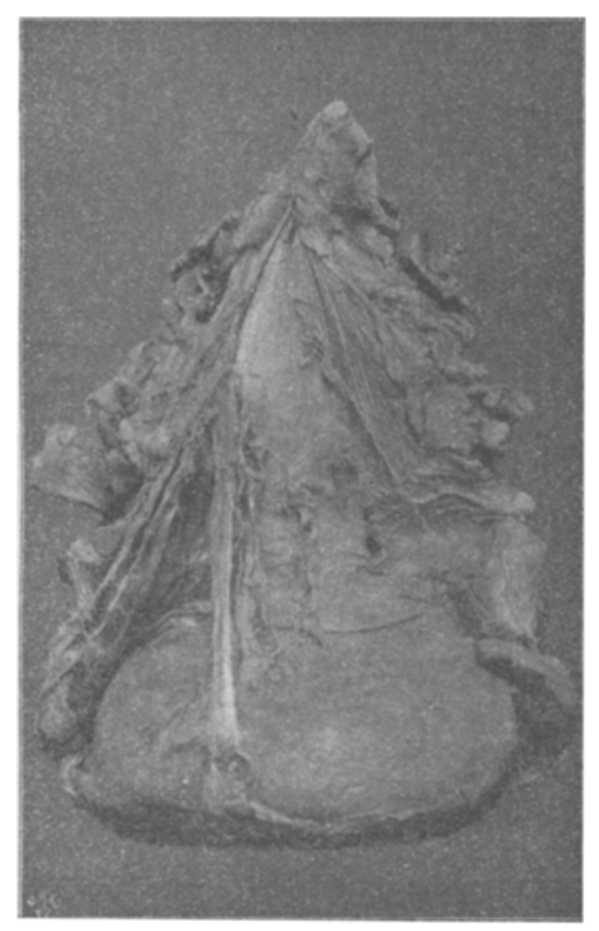

der Kopf stand in Höhe der Spinallinie. Nach Perforation leichte Fxtraction eines $3260 \mathrm{~g}$ schweren Kindes; gleich nach der Geburt folgte die Nachgelurt mit einer mässigen llenge übelriechenden, mit Kindspech vermisehten Fruchtwassers. Keine äussere Blutung.

Bei der Untersuchung fand man nun linkerseits einen penetrirenden Riss, der sich, rom Muttermund beginnend, weit nach oben erstreckte, so dass es bei Einführung der halben Hand nicht möglich war, seine obere Grenze zu erreichen. Boi der Einstellung des Risses mit Spiegeln kamen spritzende Gofässe nicht zu Gesicht; dio Ränder legten sich an einander, Därne lagen nicht vor.

Wegen des desolaten Zustandes der Entbundenen wurde von einer Laparotomie als aussichtsilos abgesehen; man begnügte sich damit, den 
sich sehlecht zusammenziohenden Uterus fest gegen die Schoussfige zu drücken und hier durch Binden festzuhalten, ferner durch subcutane Infusion ron Kochsalzlösung und Campherinjection die Herzkraft zu heben.

Die Wöchnerin ging im Laufe des Nachmittags unter zunehmenden Athmungsbeschwerden und peritonitischen Erscheinungen (hochgradiger Meteorismus, Singultus, Erbrechen) zu Grunde.

Bei der Section fand sich das Abdomen stark aufgetrieben, Magen und Därme mit Gasen gefüllt, Serosa geröthet und getrübt; in den abhängigen Partien linkerseits ca. $1 / 2$ Liter theils geronnenen, theils flüssigen Blutes; Uterusgrund fast in Nabelhöhe; die Rückenfläche desselben ist bedeckt mit bandartigen Strängen, welche besonders rechts eine bedeutende Mächtigkeit erlangen und hier vom Fundus in den Douglas herabziehen, während der Rest in verschiedener Höhe der Rückenwand inserirt (s. Abbildung). Ovarien und Tuben sind beiderseits in Pseudomembranen eingepackt.

Die obere Partie der Vorderfläche zeigt gleichfalls membranöse Auflagerungen und bedecken letztere in zusammenhängender, dicker Schicht rechterseits die Abgangsstellen von Tube und Ligamentum rotundum. Diese Membranen waren nicht ohne Einfluss auf die Entfaltung der Uterusmusculatur; denn während linkerseits die Insertion von Tube und rundem Mutterband ca. $5 \mathrm{~cm}$ von einander entfernt sind, liegen sie rechts eng aneinander; die Abgangsstelle der linken Tube steht fingerbreit höher als die rechte; die entsprechende Funduspartie ist stärker gewölbt und fällt nach rechts in flachem Bogen ab.

Der Riss verläuft links vorn seitlich gerade von oben nach unten; er beginnt ca. 2 Querfinger oberhalb des Grenzringes, durchtrennt hier nur die oberflächliche Sehicht (Peritoneum, obere Muskellamelle) weiter unten den gedehnten Abschnitt in seiner ganzen Länge, schlägt sich auf die Rückenflache der Blase über, durchtrennt aber auch hier nur den peritonealen Ueberzug, lassst die Blase selbst intact. Die Blase ist an der Stelle des Risses vom Uterus losgelöst.

Die Länge des unteren Uterinsegmentes beträgt vorn $25 \mathrm{~cm}$, hinten $16 \mathrm{~cm}$, die Länge des contrahirten Theils rorn $18 \mathrm{~cm}$, hinten $14 \mathrm{~cm}$. An der Grenze von dem gedehnten untern und den contrahirten waberen Abschnitte springt der Grenzring deutlich vor (s. Abbildung); seine Ränder umgeben eine nahezu querovale Oeffnung, deren Durchmesser 6 bezw. $4 \mathrm{~cm}$ betragen. Dieses scharfe Hervortreten des Grenzringes hebe ich besonders hervor; an keinem Präparate eines puerperalen Uterus unserer Sammlung konnte ich einen derartigen Befund, den man bei Placentarlösungen an der Lebenden so schön fühlen kann, erheben; ebensowenig erinnert sich mein Chef, Herr Director Baumm, daran, etwas Aelnliches bei Sectionen jemals gesehen zut haben. Gewöhnlich sieht man beide Abschnitte ohne scharfe Grenze in einander übergehen.

Die Musculatur des Uterus ist kräftig entwickelt; am massigsten ist dieselbe rechts vorn, wo ihre Dicke $3,5 \mathrm{~cm}$ beträgt im Gegensatz zu der voln 2,5 cm des Restes der vorderen Wand; ein nachweisbarer Dickenunterschied zwischen dieser und der der hinteren Wand besteht nicht.

Die hintere Muttermundslippe ist vollständig verstrichen; makroskopisch lässt sich hier die Grenze von Scheidenwand und Cervix nicht bestimmen; vorn dagegen grenzt sich die Cervix durch eine circuläre, 
wallartig hervortretende Erhebung, ïber welcher sich eine seichte Vertiefung findet, von der Scheide ab; rechts und links findet sich noch ein ca. $1 / 2 \mathrm{~cm}$ breiter Muttermundssaum.

Schneidet man auf die vordere Lippe ein, so zeigt sich ihr Gewebe blutig imbibirt; ihr Rand steht gut fingerbreit tiefer als der der hinteren Lippe.

Es handelt sich also in unserem Falle zweifellos um eine Schwångerschaft im retroflectirt fixirten Uterus, und wir können wohl ohne Weiteres annehmen, dass die Lageanomalie sich nicht crst in der Schwangerschaft entwickelt, sondern vor derselben bestanden hat. Schwangersehaften bei Rückwärtslagerung der Gebärmutter können verschiedenen Ausgang nehmen; entweder - und das ist wohl das glücklichste Ereigniss - tritt Spontanreposition ein, in der Regel gegen Ende des 3. Monats, wenn es nicht schon vorher zum Abort gekommen ist, oder es tritt Incarceration ein für gewöhnlich im 4. Sehwangerschaftsmonat, und endlich kann sich der Uterus bei zunehmendem Wachsthum aus dem kleinen Becken hervorheben, so dass er mit seiner vorderen Fläche über den Beckeneingang hervorragt, während ein Theil im kleinen Becken zurüickbleibt; man pflegt diesen Zustand als "partielle Retroflexion" zu bezeichnen. Auch in unserm Falle handelt es sich um letztern Zustand und finden wir denselben veranlasst durch perimetritische Verwachsungen der Rückfläche des Uterus, die eine vollständige Aufrichtung des Organs verhinderten und die hintere Wand im kleinen Becken zurückhielten. Es ist interessant zu beobachten, dass es in Folge dieser Verwachsungen nicht zum Abort gekommen ist, dass vielmehr die Adhäsionen dem Zuge des wachsenden Organs in dem Maasse nachgaben, dass die Schwangerschaft ohne Beschwerden bis zum normalen Ende verlaufen konnte. Häufig nämlich „pflegt sich der Uebergang der totalen Retroflexion in die partielle unter lästigem Harndrang und hartnäckiger Neigung zur Stukliverstopfung zu vollziehen" (Veit) ${ }^{1}$ ). Von dem Grade der in Betracht kommenden Lageabweichung und der daraus resultirenden Wachsthumsrichtung des Uterus, der Grösse des im Becken verbleibenden Uterusabschnittes und von den räumlichen Beckenverhältnissen wird es abhängen, $a b$ und wann Incarcerations-

1) Veit, Ueber die Retroflexionen der Gebärmutter in den späteren. Schwangerschaftsmonaten. Sammlung klinischer Vorträge, herausgegeben von Richard Volkmann. No. 170. 
erseheinungen auftreten; dementsprechend werden wir bei unsern Schwangeren eine Retroversio-flexio II. und III. Giades ausschliessen müssen, weil es bei diesen wohl zur Incarceration hätte kommen müssen, vielmehr sind wir genöthigt, wegen des Fehlens jeglicher Symptome von Seiten der Blase und des Mastdarms einen leichten Grad dieser Lageanomalie anzunehmen; bei diesem nämlich kann sich die Vorderfläche des Uterus frei nach oben entfalten und auch die Rückfläche wird je nach der Ausdehnung und Festigkeit etwaiger Adhäsionen dem Wachsthum des ganzen Organs nach oben in die Bauchhöhle folgen können, so dass unter günstigen Umständen nur ein kleinerer Theil des Uterus im kleinen Becken zurückbleibt, der keine Symptome zu machen braucht.

Die gleichen Verhältnisse wie bei Retroflexio uteri fixati finden sich froilich im umgekehrten Sinno bei Anteflexio uteri gravidi fixati, und ist es besonders in jetziger Zeit lehrreich und von actueller Bedeutung, den Schwangerschafts- und Geburtsverlauf nach den antefixirenden Operationen, also nach der Vesicifixur, Ventrifixur und Vaginifixur, und nach letzterer Operation besonders bei der sogenannten intraperitonealen Methode, bei der eine feste, serosofibröse Verbindung mit der Scheide hergestellt wird, zum Vergleich heranzuziehen. Bei beiden Lageanomalien entwickelt sich der Fruchthaiter, falls nicht die Adhäsionen nachgeben oder Abort cintritt, auf Kosten des nicht fixirten Theiles, bei der Retrofloxio uteri fixati auf Kosten der vorderen Wand - ich erinnere in unserem Falle an den Längenunterschied der vorderen und hinteren Wand, das abweichende Verhalten der Abgangsstellen der Eileiter und runden Mutterbänder beiderseits -- dort auf Kosten der hinteren Wand; in dem einen Falle steht der Mundermund an der Schoossfuge, in dem andern hinten am Vorberg; bei beiden Lageabweichungen wird die Schwangerschaft häufig unterbrochen, bei beiden kann es zu erheblichen Schwangerschaftsbeschwerden kommen, schliesslich sind bei beiden ernste Geburtsstörungen beobachtet; so konnte in einem von Strassmann ${ }^{1}$ ) berichteten Falle von Vaginifixatio uteri die Geburt nach Absterben des Kindes per vias naturales nicht beendet und musste Kaiserschnitt gemacht werden.

Welche Umstände nun tührten in unserem Falle zur Ruptur?

1) Strassmann, Zur Kenntniss des Sohwangerschafts- und Geburtsverlaufs bei antefixirtem Uterus. Dieses Archiy. Bd. 50 . 
Jch erwähne ausdrücklich, dass dieselbe erfolgte, bevor irgend welche Entbindungsversuche voraufgingen. Wie ersichtlich, handelt es sich um einen typischen Bandl'schen Riss, d. h. um eine Ruptur nur des unteren Segments. Die Mechanik einer solchen nun erfordert eine Fixation des Segmentes nach unten, wie sie bei Einklemmung der Muttermundslippen in Folge von räumlichen Missverhältnissen zu Stande kommt. Letztere liessen sich in unserem Falle nicht nachweisen und konnten denn auch nicht das ätiologische Moment der Fixation abgeben.

Die Hauptschuld an diesem unglücklichen Ereignisse trägt meiner Ansicht nach der durch die Dislocation bedingte veränderte Fruchtaxendruck, der im An.. fang der Geburt die fehlerhafte Stellung des Kopfes zum Beckeneingang und stärkere einseitige Dehnung des unteren Segments an der vorderen Seite zur Folgo hatte, im weiteren Verlaufe der Geburt aber zur Fixation des vorderen unteren Segmentes in weiter Ausdehnung führte.

Im Anfang einer jeden normalen Geburt steift sich (Schröder) der Uterus und lagert sich mehr an die vordere Bauchwand; seine Achse fällt annähernd mit der Achse des Beckens zusammen, steht also auf dem Beckeneingang nahezu senkrecht, und wirkt demgemäss der Fruchtachsendruck in der Verlängerung dieser Achse. In unserem Falle war der Uterus hauptsächlich rechts hinten fixirt, seine Achse verlief von rechts oben nach links unten, und in dieser Richtung bewegten nun auch im Anfang der Geburt die Wehen das Kind fort; sie drückten also den Kopf nicht auf den Beckeneingang, wie unter gewöhnlichen Verhältnissen, sondern gegen die linke vordere Wand des unteren Segments und bauchten diese vor.

Die weitere Folge davon war, dass die Erweiterung dor Cervix nicht wie bei Erstgebärenden unter normalen Verhältnissen im Beckencanal, sondern über dem Beckeneingang sich abspielte, und dass der Kopf sich schon ganz in der Cervix befand, während er noch vollständig überm Becken stand; dadurch war schon eine starke Dehnung des unteren Segmentes bedingt. "Steht aber das Ostium internum hoch über der Conjugata und begegnen dem eindrängenden Schädel Hindernisse, dann ist der Grund für einen abnormen Geburtsverlauf gelegt; „bleibt dic Cervix in der Klemme, 
dann bilden sich die Verhältnisse für die Ruptur immer mehr aus" (Bandl) $)^{1}$.

Dieser Sachverhalt lag in unserm Falle vor und muss vorgelegen haben, denn sonst hätte keine Ruptur der geschilderten Art entstehen können. Die Art und Weise nun, auf welche die Einklemmung der Muttermundslippen zu Stande kam, war eine ungewöhnliche; an derselben war nicht ein Missverhältniss zwischen Kopf und Becken schuld, sondern sie wurde veranlasst durch das Andrängen des Kopfes an die vordere Beckenwand, gegen welche der Fruchtachsendruck, wie leicht verständlich, auch nach der Lagecorrectur und nach Eintreten des Kopfes infolge der hinteren Fixation des Uterus wirken musste. Wäre der Kopf gleich im Anfange der Geburt über dem Beckeneingang festgehalten worden, zu einer Zeit, in welcher das untere Segment noch nicht überdehnt war, so hätte dieses Hinderniss vielleicht überwunden werden können; das untere Segment hätte sich vermöge der ihm innewohnenden Elasticität über den Kopf zurückgezogen; jetzt aber im Zustand der Ueberdehnung und hochgradig verminderten Retractionsfähigkeit war dieses Anpressen und Herunterzerren der Muttermundslippe durch den Kopf verhängnissvoll.

Dass dem so ist, beweist mir auch der Tiefstand der vorderen Lippe am Präparat. Bei der Aufnahme befand sich der Nuttermund fingerbreit über der Schoossfuge. Nach dem Eindrücken des Kopfes auf den Beckeneingang ging nun die Erweiterung des Muttermundes derart vor sich, dass die hintere Lippo im Bogen um den eintretenden Kopf nach hinten und oben zurückgezogen wurde, und wird nun bei dem anfänglichen Tiefertreten desselben auch ein Zug an der verderen Lippe ausgeübt und diese iu den Bereich der hinteren Fläche der vorderen Beckenwand gezogen worden sein, wo sie eben dann von dem gegen die Wand andrängenden Kopf gefasst und an ihr entlang nach unten geschoben wurde.

Erwähnte Umstände allein würden sicher genügen, das Eintreten der Ruptur zu erklären; als weiteres Moment kommt noch die veränderte Beschaffenheit des unteren Segments hinzu, das eben dadurch, dass sich der Fruchthalter hauptsächlich auf Kosten

1) cf. Herrmann W. Freund, Die Mechanik and Therapie der Uterusund Scheidengewölberisse. Zeitschrift f. Geburtsh. u. Gynäk. Bd. XXIII. S. 444 . 
der vorderen Wand entwickelte, vielleicht zur Ruptur mehr disponirt war, als ein normales unteres Uterinsegment. Es lässt sich diese Prädisposition schwer beurtheilen, noch schwerer beweisen, ganz von der Hand zu weisen ist sie aber nicht, wenn ihr auch neben den oben erwähnten, die Ruptur begünstigenden Momenten eine untergeordnete Bedeutung zukommt.

Zum Schlusse ist es mir eine angenehme Pflicht, meinem hochverehrten Chef, Herrn Director Baumm, für die gütige Ueberlassung des Falles meinen besten Dank auszusprechen. 